\title{
CARACTERIZACIÓN DE LAS \\ COOPERATIVAS DE TRABAJO ASOCIADO EN BUCARAMANGA, (COL)
}

\author{
Johanna Sarit Riaño Acosta \\ Maestría en Hermenéutica Jurídica (en curso). Abogada Universidad Santo Tomás, \\ Seccional Bucaramanga (Col); Docente Universidad Facultad de Derecho \\ Universidad Santo Tomás Seccional Bucaramanga (Col). \\ E-mail: johanasarit@gmail.com
}

\begin{abstract}
Resumen
El siguiente artículo incursiona en el concepto de economía solidaria con el fin de acercarse a la idea de Cooperativas de Trabajo Asociado CTA en Colombia; crea un referente teórico, histórico y legal sobre su desarrollo en la ciudad de Bucaramanga y el departamento de Santander. La investigación elabora instrumentos de recolección de información que dan cuenta del estado en que se encuentran los miembros de las CTA en la ciudad de Bucaramanga y de esta forma abre el debate sobre la proliferación de estas Cooperativas con el fin de vincular mano de obra a un bajo precio, situación que vulnera los derechos del trabajador, así el autor de este texto se enfrenta a una dicotomía al observar como estas cooperativas ejercen sus labores con el consentimiento de sus miembros y a la vez como se ven vulnerados principios laborales como el de la realidad sobre la forma, el de favorabilidad y el de legalidad. Finalmente el texto advierte sobre la naturaleza de las CTA, pues una es la forma como se encuentran registradas en la Cámara de Comercio de la ciudad y otra las funciones que desempeñan.
\end{abstract}

\section{Palabras clave}

Cooperativas de Trabajo Asociado, economía solidaria, trabajadores, condiciones laborales, principios laborales y vulneración de derechos.

\begin{abstract}
The following paper immerses in the concept of solidary economy with the objective to approach the idea of Cooperative of Associated Work (CAW) in Colombia. It creates a theoretical, historical and legal reference about its development in the city of Bucaramanga and the Department of Santander. This research elaborates information recollection instruments that shows the state in which the members of the CAW in the city of Bucaramanga, and with this way, to open the debate about the proliferation of the Cooperatives with the objective to hire workers at low prices, situation that affect the rights of the workers. The author of this text faces a disjunctive while observing how the cooperative exercise their work with the consent of their members and at the same time see how the labor principles are violated such as "prevalence of reality over formalism, favorability, and legality". Finally, the paper warns about the nature of the CAW, which is a way how they are found registered in the City's Commerce Chamber and other functions they may perform.
\end{abstract}

\section{Key Words}

Associated Work Cooperatives, solidarity economy, workers, working conditions, labor principles and rights violations. 

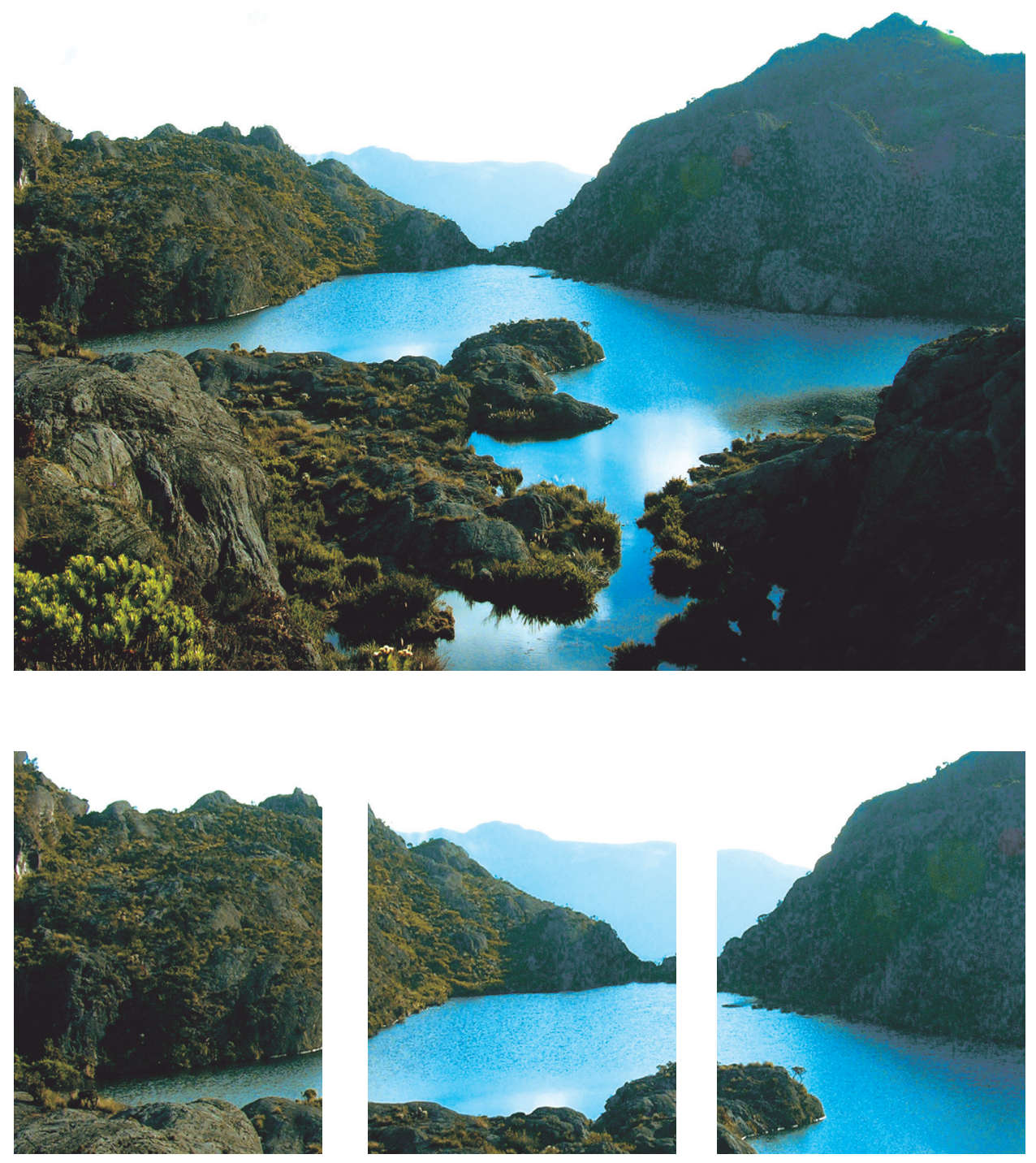

Laguna "La Negrita" 


\section{CARACTERIZACIÓN DE LAS \\ COOPERATIVAS DE TRABAJO ASOCIADO EN BUCARAMANGA, (COL)*}

Johanna Sarit Riaño Acosta

Para el año 2007, según información de la Superintendencia de Economía Solidaria, existían en el país, entre Cooperativas y Precooperativas de Trabajo Asociado alrededor de 12.059 organizaciones, de las cuales el departamento de Santander aportaba el $10.33 \%$, destacándose su participación nacional junto con el departamento de Valle del Cauca y el distrito capital Bogotá (APÉNDICE 1). No obstante, a pesar de la considerable agregación de empleo que este tipo de organizaciones realiza, existen dudas acerca de su oportunidad en materia de dignidad y desarrollo laboral. Un estudio de la Superintendencia de Economía Solidaria (2003), encontró que de una muestra de 162 Cooperativas de Trabajo Asociado solo el 25\% operaba legalmente, el 51\% funcionaba como Empresas de Servicios Temporales y el 8\% como agrupadora en salud. Este fenómeno ha creado una gran polémica alrededor de la naturaleza de esta clase de cooperativas y su objeto social en el ámbito laboral.

Ante la notable participación del departamento en lo referente a las CTA se evidencia que más del $50 \%$ operan en Bucaramanga, surgió la inquietud de conocer el funcionamiento real de las mismas en el plano local, evaluar su eficacia con respecto a lo dispuesto por la normatividad que la regula, así como con los principios en materia laboral del Estado Social de Derecho Colombiano que lo enmarca.

Este artículo es producto de la Investigación Impacto de las Cooperativas de Trabajo Asociado en Bucaramanga en la generación de empleo y desarrollo, del Grupo de Investigación Neoconstitucionalismo y Derecho, Línea Neoconstitucionalismo y Convivencia Pacífica. Facultad de Derecho - Universidad Santo Tomás Seccional Bucaramanga. Auxiliares de Investigación: Juan Pablo Romero Correa; Mónica Gómez Villalba; Yuberly Chacón Ardila, Eluz Pierina Solano Amaya. 


\section{Cooperativismo: una alternativa para el desarrollo económico y social}

En una época en la cual la economía ha sido catalogada como disciplina poco ética e inhumana, debido a los agudos efectos causados por las crisis de los modelos de desarrollo emergentes y la escasa recuperación de los mismos, especialmente en los sectores deprimidos de la sociedad de Latinoamérica, surge una propuesta innovadora que pretende subsanar los efectos regresivos en materia de empleo: la denominada economía solidarial.

Según Razeto (1997) la economía y la solidaridad pueden converger en una escuela de pensamiento según la cual

"se recupere la economía como espacio de realización y actuación de los valores, (...) desarrolle un proceso interno que le abra espacios de reconocimiento y actuación al valor de la solidaridad (...) que opere y actúe en las diversas fases del ciclo económico." (p, 5).

En Colombia, la economía solidaria se ha expresado en forma de organizaciones de consumo, producción, servicios y asociación con base en el cooperativismo, el sentimiento de ayuda mutua y reciprocidad. Existen antecedentes del cooperativismo desde la conformación de la Nación misma ${ }^{2}$, no obstante, su desarrollo sobresale desde 1929, época en que se establece como estrategia gubernamental para enfrentar la crisis económica, alentada por el Partido Socialista Revolucionario.

Las primeras cooperativas aparecieron en Medellín, Bogotá y en menor proporción en Bucaramanga ${ }^{3}$. No obstante, es importante señalar la relevancia que tuvo el desarrollo del cooperativismo en Santander, desde los puntos de vista económico y social, puesto que éste se desplegó de manera diferente que en el resto del país, al construirse alrededor de organizaciones campesinas y comunitarias de base, gestadas en el sur del departamento, en las provincias Guanentina, Comunera y Veleña, desde la década de los sesenta. Este fenómeno contrasta con el proceso de cambio económico mundial orientado a consolidar un modelo paternalista, que apoyaba la producción interna especialmente la industrial, el denominado Estado de Bienestar impulsado por la Comisión Económica Para América Latina (CEPAL).

Uno de los principales gestores de este proceso fue el Padre Ramón González Parra, quien al evidenciar las necesidades económicas y sociales que vivían los

1 Entre los principales exponentes en América Latina de la Economía Solidaria se encuentra el chileno Manfred Max Neef.

2 Para mayor profundidad en antecedentes históricos del cooperativismo revisar reseñas de organizaciones como Fempopular, Portal Cooperativo, entre otros.

3 En 1933 se organizaron las cuatro primeras cooperativas: Empleados de Bogotá de buses de Santa Fe Ltda., Cundinamarquesa de miel y panela, Cooperativa de consumo de empleados y obreros de la fábrica de Cementos Diamante; de ahí comenzó su expansión, impulsada por las reformas impulsadas por el Presidente Alfonso López Pumarejo, de 296 cooperativas en 1947 se evidencian 410 cooperativas en 1957 y de 68.741 a 175.510 socios en el mismo periodo. 
campesinos marginados del sur del departamento, así como su abandono o no inclusión en los planes económicos estatales, propuso un esquema denominado Desarrollo hacia adentro ${ }^{4}$, que apoyó los procesos productivos agrícolas (inicialmente) de la región basado en la unión de intereses, las relaciones de confianza y de reciprocidad existentes entre la población. Las primeras cooperativas en la zona se dedicaron a proveer servicios de ahorro y crédito. El resultado de este proceso fue la fundación de la Cooperativa de ahorro y crédito COOPCENTRAL en 1964, la creación de la Asociación de Organizaciones Campesinas y Populares de Colombia (El Común), la Emisora Comunitaria La Cometa, y la Federación Nacional de Tabacaleros (Fedetabaco), entre otros movimientos de representación popular comunitaria. Este proceso gestado implicó todo un crecimiento cooperativo concatenado que mejoró la calidad de vida y el desarrollo socioeconómico de la región ${ }^{5}$.

Guerrero (2005) sintetiza la historia cooperativa santandereana argumentando que durante el periodo de 1930 a 1990 se fundaron en Santander un total de 640 cooperativas, las cuales generaron a finales de los años ochenta 4620 empleos directos con 139.437 asociados (Guerrero, 2005, p. 319). En la década de los cuarenta, se pactaron en las convenciones colectivas, subsidios a las cooperativas, y permisos sindicales a los dirigentes cooperativos. Más tarde, en los años cincuenta se sentaron las bases para el desarrollo cooperativo y así en los pliegos de peticiones, se solicitaba el apoyo para la creación de fondos de ayuda mutua con diferentes servicios; un ejemplo de ello es el caso de la Panadería Trillos y Trefilco S.A, donde se creó el sistema uno a uno: el trabajador aportaba cien pesos (\$100) y el empleador otros cien pesos $(\$ 100)$, creando un hábito que llevó a la posterior fundación de las cooperativas, la creación de cajas de ayuda mutua y fondos cooperativos. En la década de los setenta se crearon más cooperativas de trabajadores rurales y urbanos, siempre se buscaba mejorar las condiciones sociales y económicas de los mismos.

\section{Cooperativas de Trabajo Asociado: Concepto y Configuración Legal}

Una forma particular de cooperativismo ha sido las dedicadas al Trabajo Asociado, estas fueron reconocidas normativamente por el Decreto 2059 de 1968, el cual estableció el concepto de cooperativismo, al vincular la satisfacción de las necesidades sociales y económicas de los asociados; más tarde, mediante el Decreto 1613 de 1971, se autorizó la creación de las dependencias regionales de

4 Basado en los conocimientos sobre economía solidaria adquiridos en sus estudios en Francia y Canadá, y la influencia de la Iglesia Católica donde se desempeñaba como Director del Secretariado Diocesano de Pastoral Social (SEPAS) en San Gil. Según Buchelli (2001) "la idea del Padre Ramón fue la de crear un Movimiento Social Comunitario que en su trabajo de grado tituló como: Proyecto de cambio y desarrollo rural en la Diócesis de Socorro y San Gil".

5 Esta experiencia santandereana alrededor de la economía solidaria ha sido resaltada por organizaciones como el Programa de Naciones Unidas para el Desarrollo (PNUD) por sus efectos en el desarrollo económico -social que sirvió como atenuante del conflicto armado. 
la Superintendencia de Cooperativas que posteriormente fueron modificadas por la Ley 24 de 1981, la cual transformó la Superintendencia en el Departamento Administrativo Nacional de Cooperativas (DANCOOP); esta ley fue reglamentada mediante el Decreto 3559 del mismo año.

El 23 de Diciembre de 1988, la Ley 79, actualizó el régimen legal de cooperativismo, el cual estableció la autonomía que debían tener las cooperativas, su interés social sin ánimo de lucro, el ingreso y el retiro voluntario e ilimitado basado en la democracia con actividades de educación cooperativa, su integración económica y social, y la igualdad de derechos de los socios, sin tener en cuenta sus aportes y la legislación que regía los trabajadores dependientes.

Con el Decreto 0468 de 1990, se fijaron las características de las Cooperativas de Trabajo Asociado (CTA), el número mínimo de asociados que no podía exceder de 10 , la voluntariedad al ingreso y retiro, su autonomía, su régimen de compensaciones, y las obligaciones del ISS y cajas de compensación en su afiliación. Seguidamente, el Decreto 3081 de 1990, estableció el límite en que podían reajustarse los aportes sociales que realizaban los asociados en las cooperativas, pre-cooperativas y empresas de servicios.

Luego se profiere la Ley 454 de 1998, que crea la Superintendencia de Economía Solidaria y el Fondo de Garantías para las Cooperativas Financieras, de Ahorro y Crédito, que fueron reglamentadas por el Decreto 2206 de 1998. Mediante el Decreto 510 de 1999, se dictaron disposiciones sobre el sistema financiero que transformaron las instituciones financieras de naturaleza cooperativa en sociedades comerciales. En el mismo año el Decreto 1401, desarrolló la estructura y funciones de la Superintendencia de la economía solidaria y finalmente la Ley 759 de 2002, ajustó las normas del estatuto orgánico financiero.

En la actualidad las CTA se rigen por el Decreto 4588 de $2006^{6}$, el cual dispone los lineamientos básicos sobre la organización y funcionamiento de las cooperativas y pre- cooperativas, la forma para contratar con terceros, y aclara las prohibiciones y obligaciones de los asociados. Posteriormente la Ley 1233 de 2008, fortalece su control y crea las contribuciones especiales a su cargo con destino al SENA, ICBF y cajas de compensación.

Todo este marco normativo, especialmente el emergente después de la Constitución de 1991 en la cual el derecho al trabajo se sitúa en el ámbito fundamental. De conformidad a lo estipulado en el parágrafo primero del artículo 6 del Pacto Internacional de Derechos Políticos Económicos, Sociales y Culturales, el derecho al trabajo es aquel que tiene toda persona de ganarse la vida mediante una labor libremente escogida o aceptada. El constituyente impone al Estado la obligación de proteger de manera permanente las fuentes de trabajo, crea condiciones favorables al libre ejercicio de la actividad económica, salvaguarda a los más débiles, asegura

$6 \quad$ Derogado por el Decreto 468 de 1990 
un mínimo vital al trabajador y establece límites a la autonomía de las partes que constituyen las condiciones de trabajo, por ello, el desarrollo legislativo de este derecho fundamental se concreta por mandato del artículo 53 de la Carta Magna, en los siguientes principios:

1. Igualdad de oportunidades

2. Remuneración mínima, vital y móvil, proporcional a la cantidad y calidad del trabajo

3. Estabilidad en el empleo

4. Irrenunciabilidad de los beneficios mínimos legalmente establecidos

5. Transacción y conciliación sobre derechos inciertos y discutibles

6. Favorabilidad

7. Primacía de la realidad

8. Garantía de la seguridad social, capacitación, adiestramiento y descanso

9. Protección especial a la mujer, a la maternidad y al trabajador menor de edad.

En lo que respecta a la vinculación contractual, se ejerce una figura distinta a la relación laboral común existente entre empleador y trabajador, debido a que poseen reglamentos internos que generan la renuncia a la protección especial que les otorga la normatividad, en este caso el Código Sustantivo de Trabajo.

Las CTA poseen características especiales al realizar sus actividades basándose en el principio de autonomía, es decir, se toma en cuenta la actividad económica de la cooperativa y de acuerdo a esta, se organiza y se establece las condiciones de trabajo bajo las cuales están cobijados sus asociados. De acuerdo con lo anterior, dentro de sus posibilidades para contratar, existe el convenio o avenencia con terceros a los cuales las CTA les ofrecen bienes o servicios, sin necesidad de que exista la subordinación o dependencia para no atentar contra la autonomía propia de estas. No obstante, las CTA no podrán actuar como empresas de intermediación laboral, ni disponer del trabajo de los asociados para suministrar su mano de obra temporal a usuarios o a terceros beneficiarios; tampoco podrán permitir que respecto de los asociados se generen relaciones de subordinación o dependencia con terceros contratantes; cuando se configuren prácticas de intermediación laboral o actividades propias de las Empresas de Servicios Temporales (EST), el tercero contratante, la CTA y sus directivos, serán solidariamente responsables por las obligaciones económicas que se causen a favor del trabajador asociado; tampoco actuarán como asociaciones o como agremiaciones para la afiliación colectiva de trabajadores independientes al Sistema de Seguridad Social, ni como asociaciones mutuales para los mismos efectos.

Asimismo, al poseer ciertas particularidades por su unión voluntaria de personas con objetivos, finalidades y relaciones en común, adquieren los derechos como propietarios o poseedores de los medios de producción y constituyen una estructura organizada dirigida hacia una acción colectiva, que genera alternativas y mecanismos de inserción económica para muchos trabajadores, razones que justifican de la mano 
con su objeto social, la intervención de Estado para buscar medios que ayuden a incentivar y apoyen su inclusión en el sector de la economía, de ahí la decisión estatal de otorgarles garantías como excepciones tributarias y el pago a mínima escala de las obligaciones parafiscales, con el propósito de generar oportunidades de vinculación laboral a aquellos que no poseen los medios suficientes para crear sus propias fuentes de subsistencia y bienestar (Gómez, 2008).

\section{MÉTODO}

El alcance que la presente investigación se propuso fue descriptivo de tipo transversal, con un enfoque mixto, valiéndose de técnicas especialmente cuantitativas que emergen del uso de categorías de un análisis de la institucionalidad de las CTA en Colombia y especialmente su marco legal. La herramienta de recolección de datos idónea para tal fin se constituyó en la encuesta estructurada.

Una caracterización inicial de las CTA existentes en Santander, da cuenta que su mayoría se encuentran ubicadas en Bucaramanga $(51,12 \%)$, seguida de Barrancabermeja con 10,35\%. El Área Metropolitana de Bucaramanga agrupa la mayor parte de los trabajadores asociados por medio de cooperativas pues en total los cuatro municipios del área aportan aproximadamente el 63\% del total departamental (APÉNDICE 1). En contraste, según la base de datos de la Cámara de Comercio de Bucaramanga para 2007, existían registradas aproximadamente 992 CTA, de las cuales 638 tienen su sede en Bucaramanga. De acuerdo a este número, para el análisis de las CTA en la ciudad de Bucaramanga, se practicaron 301 encuestas dirigidas a los asociados y 34 encuestas a los representantes legales de las Cooperativas de Trabajo Asociado, se aplicó un muestreo aleatorio simple con un error de 5,7\%, sobre el 50\% de la población total.

\section{RESULTADOS}

Del número total de encuestas efectuadas a los representantes legales de las CTA, se encontró que un $12 \%$ corresponde a pre-cooperativas y un $88 \%$ a Cooperativas lo que indica, de acuerdo al proceso de formación de las cooperativas, que existe una marcada tendencia a la fundación de tales organizaciones en Bucaramanga.

Cuando se preguntó a los asociados, acerca de la facultad de vinculación; se encontró que el mayor porcentaje de los asociados la realizó de manera voluntaria, en un porcentaje menor señalaron que se hacía de manera obligatoria, vinculación que se realiza por parte de las empresas a partir de la creación de cooperativas o precooperativas para que sus empleados se conviertan en asociados; otra proporción menor no contestó. 
Figura 1. Voluntariedad de la vinculación

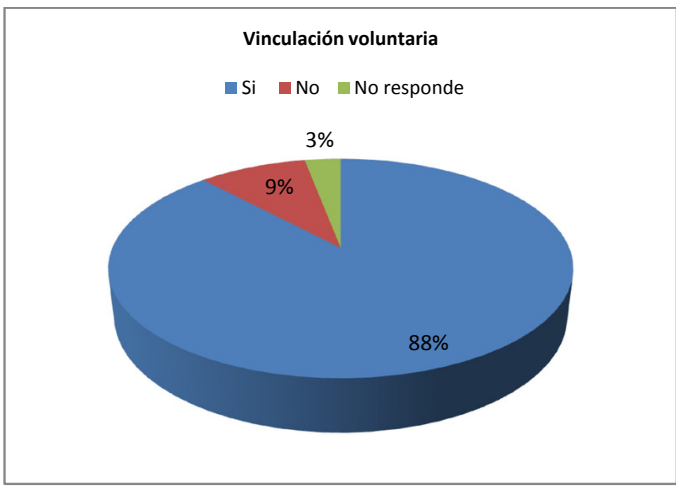

Fuente: la autora

En lo que respecta a la edad del personal que se encuentra en calidad de asociado, al efectuar las encuestas a los representantes legales de las CTA, se observó que las cooperativas de Bucaramanga tienen un $82 \%$ como asociados a mayores de edad y solo un $12 \%$ cuenta con menores entre 16 y 17 años; el $6 \%$ restante no se pudo determinar.

Figura 2. Edad del asociado

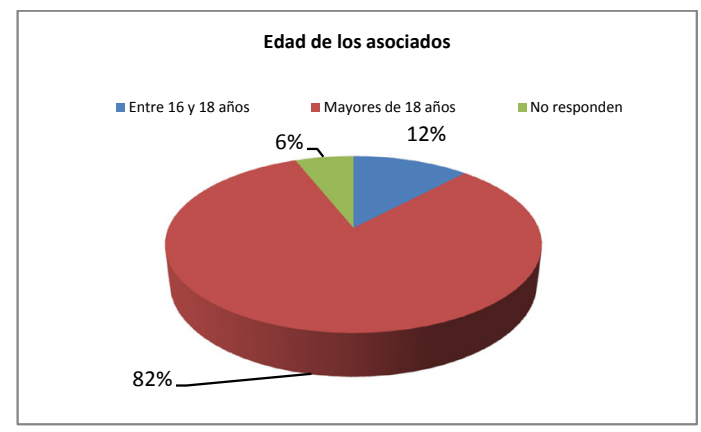

Fuente: la autora

Al cuestionarse a los asociados frente al origen de las órdenes en el desarrollo de sus actividades, se encontró que la mayor parte de los asociados manifestó recibir órdenes de la empresa en mayor medida, así como también del Representante legal, 
los coordinadores de las pre-cooperativas y las Cooperativas, en general. Es de anotar que una proporción menor pero no insignificante, manifestó que no existía tal subordinación.

Figura 3. Órdenes recibidas o delegadas

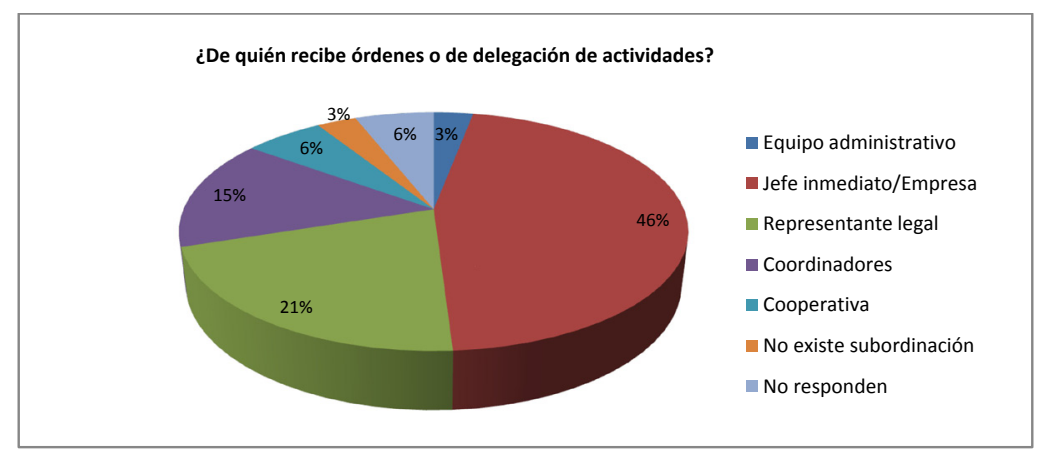

Fuente: la autora

Sobre la fijación del horario de trabajo, del mismo modo se puede constatar, que este es fijado en un 50\% de los trabajadores asociados por parte de la empresa contratante, un $29 \%$ se establece a través del régimen compensatorio de trabajo, el resto se encuentra repartido entre un acuerdo de las partes, la Cooperativa y de manera directa por cada clase de contrato que se suscriba. Lo cual enuncia un claro componente de dependencia y/o subordinación entre las partes, contrario a los principios cooperativos.

Figura 4. Horario de los asociados

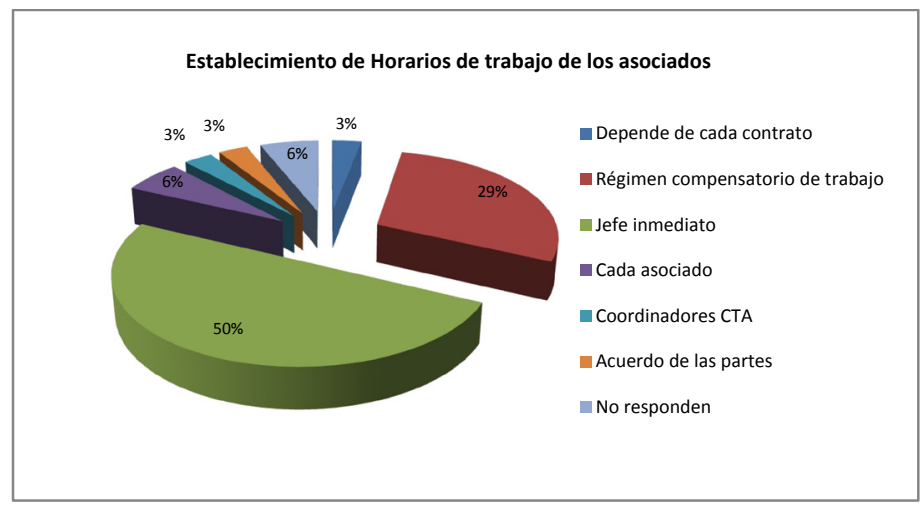

Fuente: la autora 
Frente a los materiales para la prestación del servicio, es importante señalar que el $89 \%$, lo suministra la empresa en donde se realiza la prestación; sólo un porcentaje mínimo manifestó que lo suministraba la cooperativa o pre-cooperativa, según el caso, es decir, aquellos no son dueños de los medios de producción sino de su capacidad laboral.

Al preguntarse si habían estado afiliados a alguna cooperativa anteriormente, se observa que un alto porcentaje se ha encontrado vinculado a cooperativas de trabajo asociado. Sólo un 15\% contestó que no, este reporte confirma la tendencia a buscar una fuente de sostenimiento a través de las CTA, y el gran impacto que las cooperativas de trabajo asociado han presentado en el panorama del sector productivo en Bucaramanga. De ese porcentaje de asociados se evidenció que su actividad laboral la desempeñan en sectores de servicios varios, ello permite determinar la demanda de este tipo de servicios en el sector productivo bumangués, lo siguen en su orden el sector salud, el sector educativo, vigilancia y confecciones, alimentos, aseo y asesoría jurídica.

Figura 5. Tipos de cooperativas

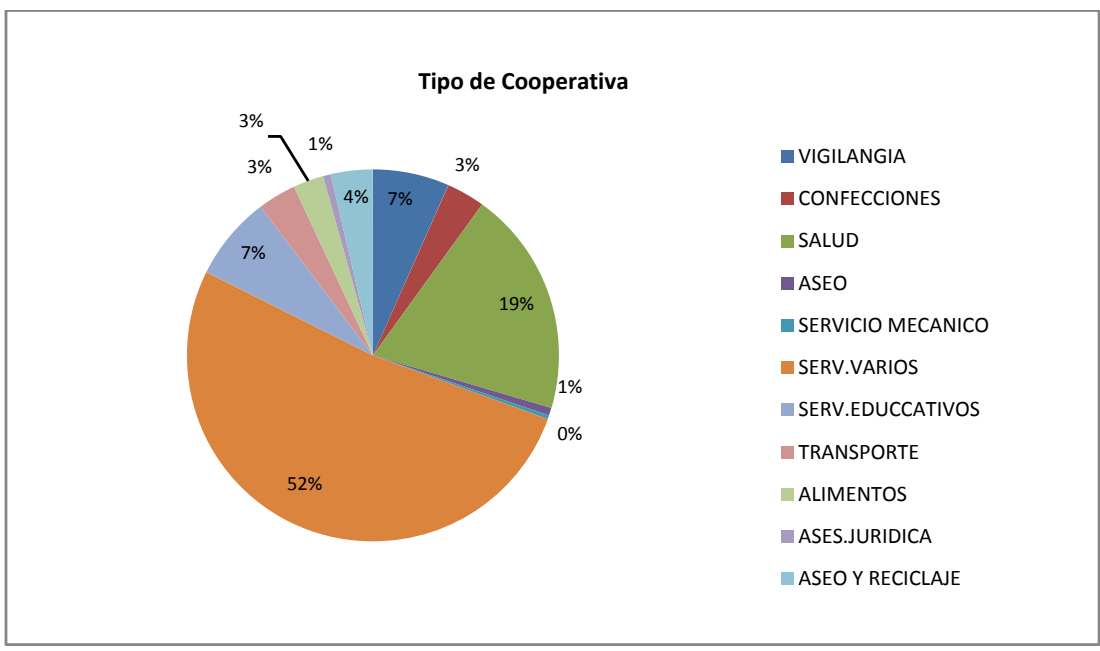

Fuente: la autora

En lo que respecta a la permanencia en la cooperativa, existe un porcentaje repartido entre aquellos que tienen al menos de un año en la Cooperativa y los que cuentan con dos y cinco años, por lo que no se podría afirmar que no existe inestabilidad en la actividad de asociación por parte de sus miembros. 
Figura 6. Fecha de Vinculación

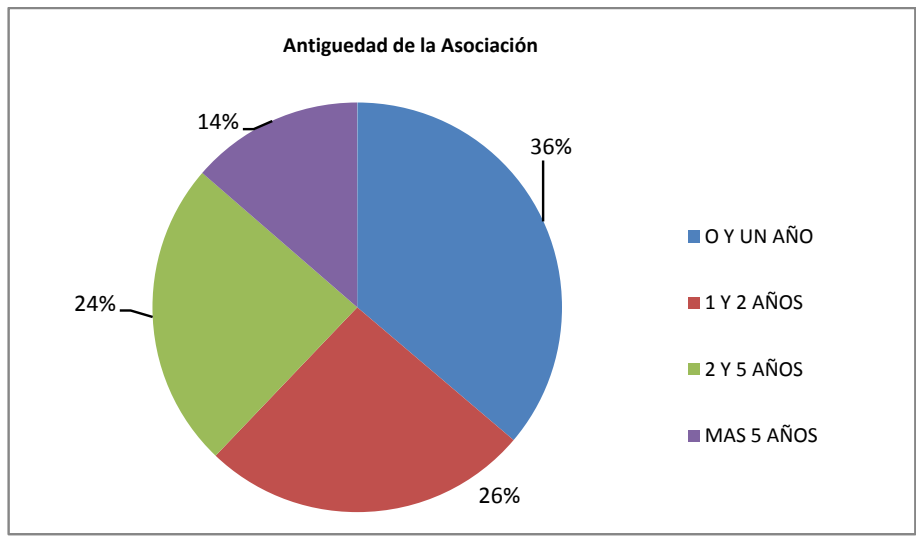

Fuente: la autora

En la creación de la Cooperativa, se observó que la mayor parte de los asociados no participaron en su conformación, sólo un 10\% afirmó su participación, lo cual expresa que la vinculación del asociado se presenta una vez creada la cooperativa, por decisión unilateral de las empresas que involucran a sus empleados en una nueva forma de asociación, otro elemento contrario a su esencia legal.

Figura 7. Participación en la constitución de la cooperativa

PARTICIPACIÓN EN LA CONSTITUCIÓN

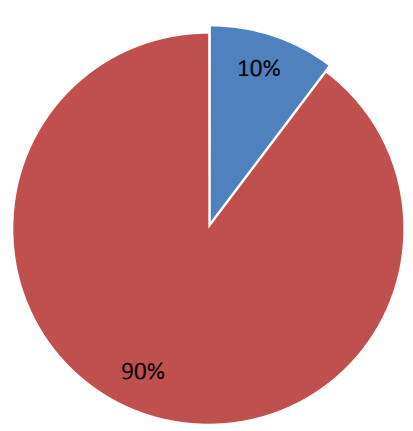

- SI NO

Fuente: la autora 
Este resultado contrasta con el modo de participación de los asociados en la toma de decisiones. Se encontró que una proporción significativa no participa en la toma de decisiones, esto es el $47 \%$, en este punto quienes deciden son las directivas de la cooperativa. Para aquellos que manifestaron participar en la toma de decisiones, la gran mayoría lo han hecho para elección de directivos en un $43 \%$, en elección del represente legal, el 23\%, en aspectos financieros el 17\% y en ampliación de la cooperativa, un $15 \%$. Lo anterior señala la ausencia de una participación equitativa en la toma de decisiones por parte de los cooperados.

Figura 8. Participación en la toma de decisiones

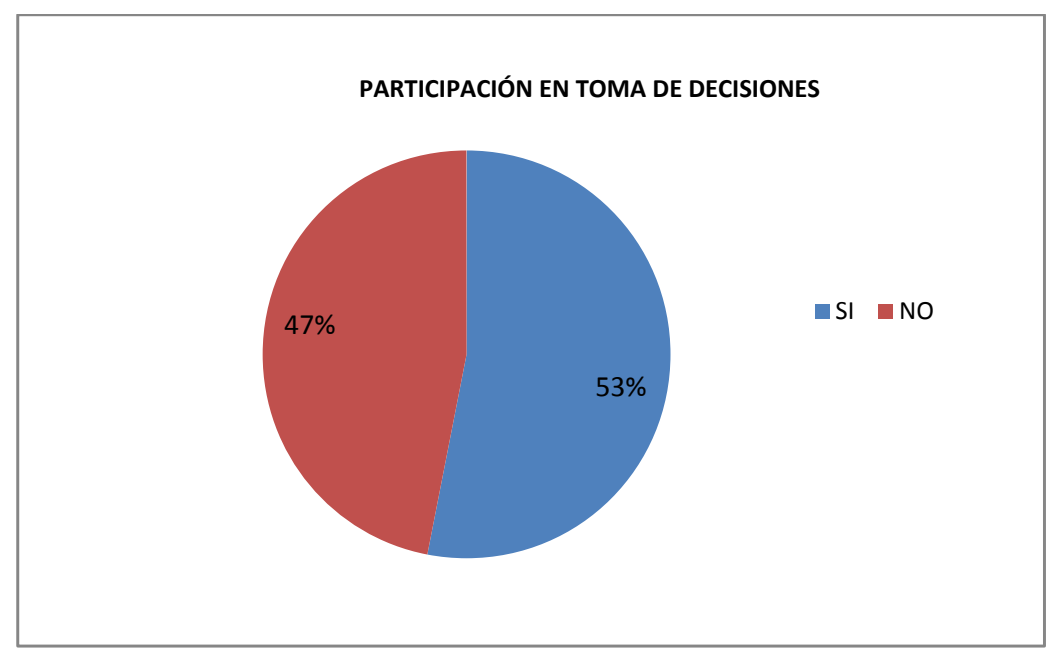

Fuente: la autora

A aquellos asociados que manifestaron no participar en la toma de decisiones de la Cooperativa a la cual pertenecían, se les preguntó: ¿En quién o quiénes radica el poder de decisión o la toma de decisiones en la cooperativa? Se encontró en sus respuestas que este corresponde al cuerpo directivo, lo cual se evidencia el desconocimiento acerca de la estructura misma de las Cooperativas. Muchas decisiones no son consultadas por los cooperados y en otras oportunidades el asociado no conoce exactamente cómo es el verdadero funcionamiento y sentido de las cooperativas en la toma de decisiones. 
Figura 9 y Figura 10. Poder de decisión
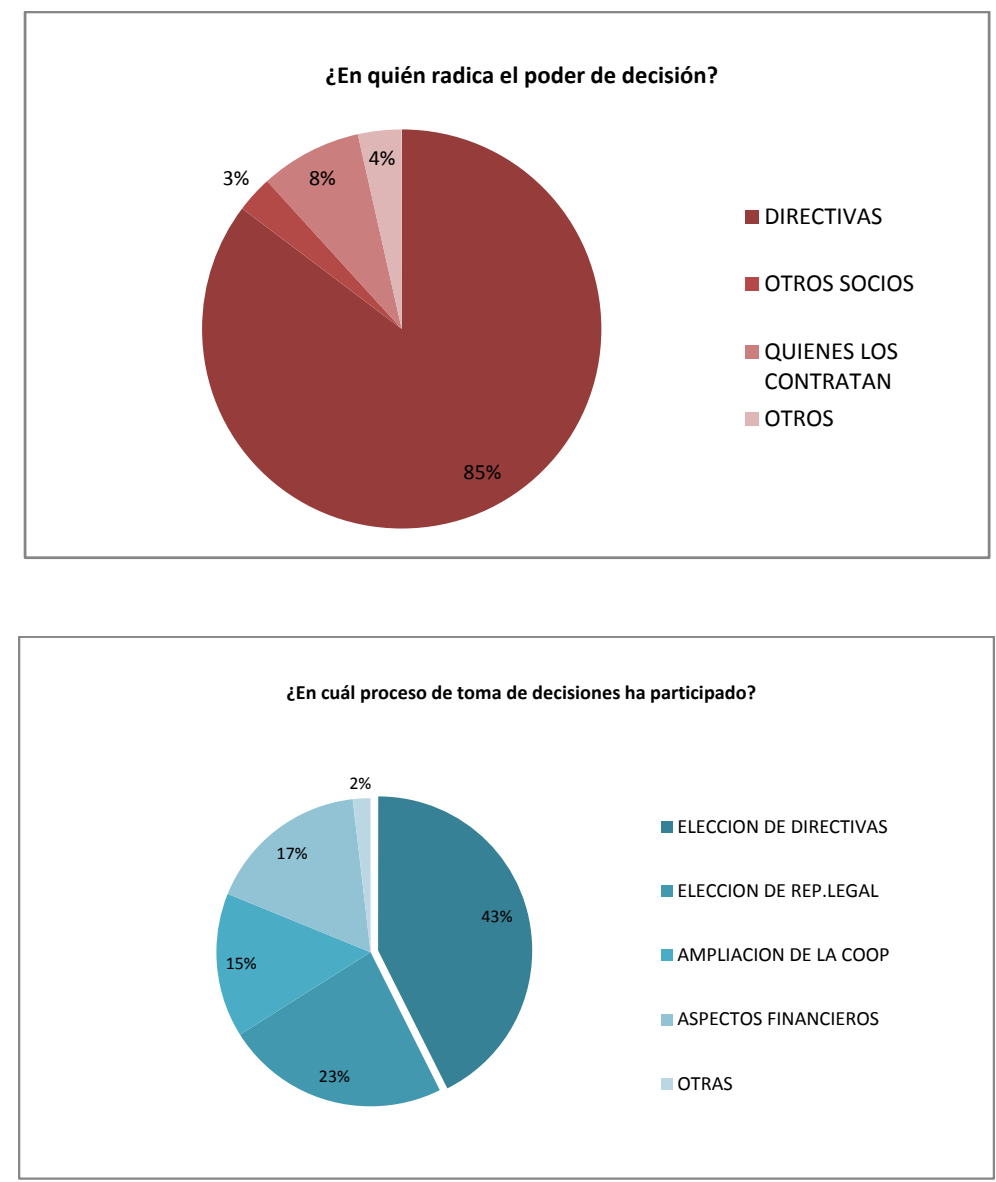

Fuente: la autora

La participación del asociado es muy escasa en la constitución de pre-cooperativas, de hecho los asociados prácticamente llegan con posterioridad a su constitución y en muchas oportunidades constituye un requisito que exige la empresa en donde van a prestar sus servicios para que puedan acceder a dicha prestación.

La participación de los asociados en muchas ocasiones se limita a la elección de directivas, algunas cuestiones financieras y ampliación de las cooperativas, sin embargo, se puede concluir que existe un desconocimiento de varias cuestiones de gran importancia para el sector cooperativo y que incumbe a los asociados directamente. 
Figura 11 y Figura 12. Participación en la elección de directivas
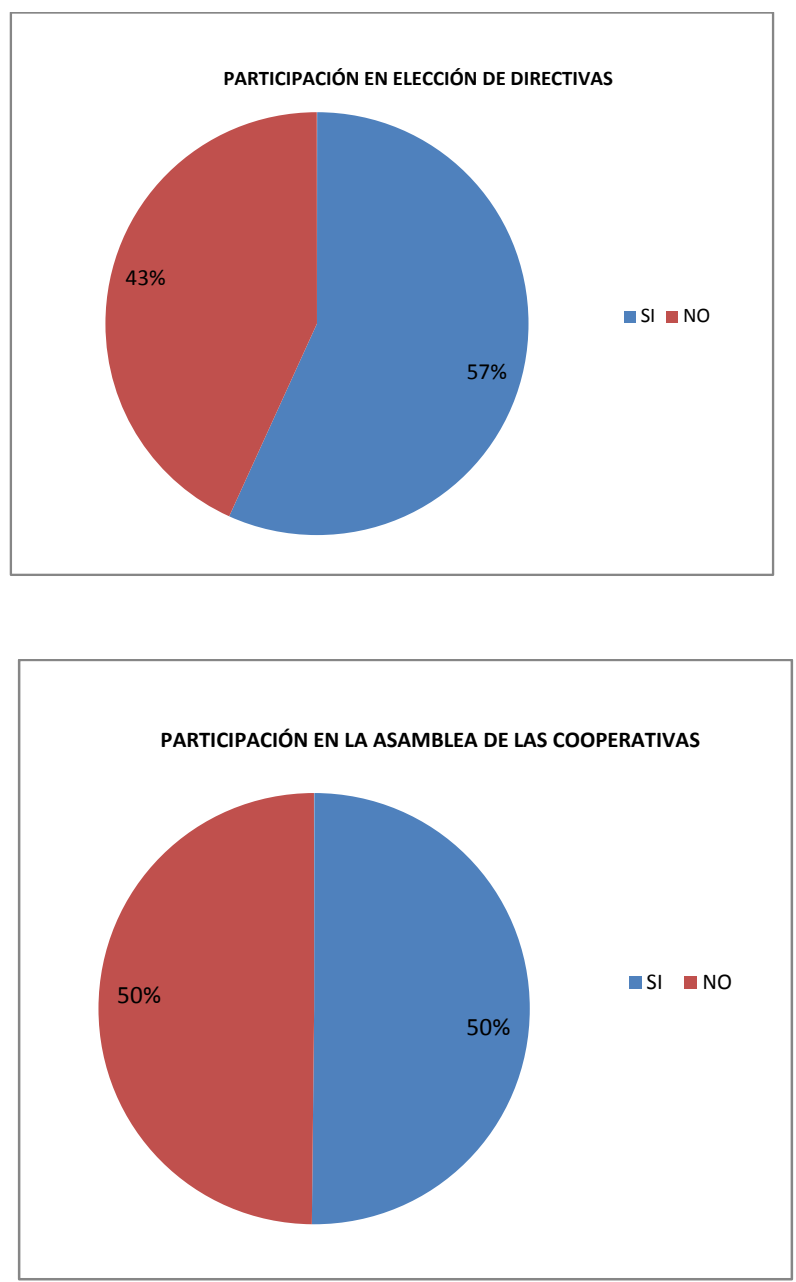

Fuente: la autora

Si bien la mayor parte de los asociados encuestados manifestó conocer los estatutos de la cooperativa a la cual se encuentra vinculado, a pesar de esto no se evidencia claridad sobre el modo de ejercer y tomar las decisiones, así como del modo de fijación de horarios. Pareciera que el carácter de asociado no es muy claro para sus integrantes, que si bien se denominan así no alcanzan a evidenciar los beneficios que ello implica. 
Figura 13 y Figura 14. Conocimiento de los Estatutos de la Cooperativa
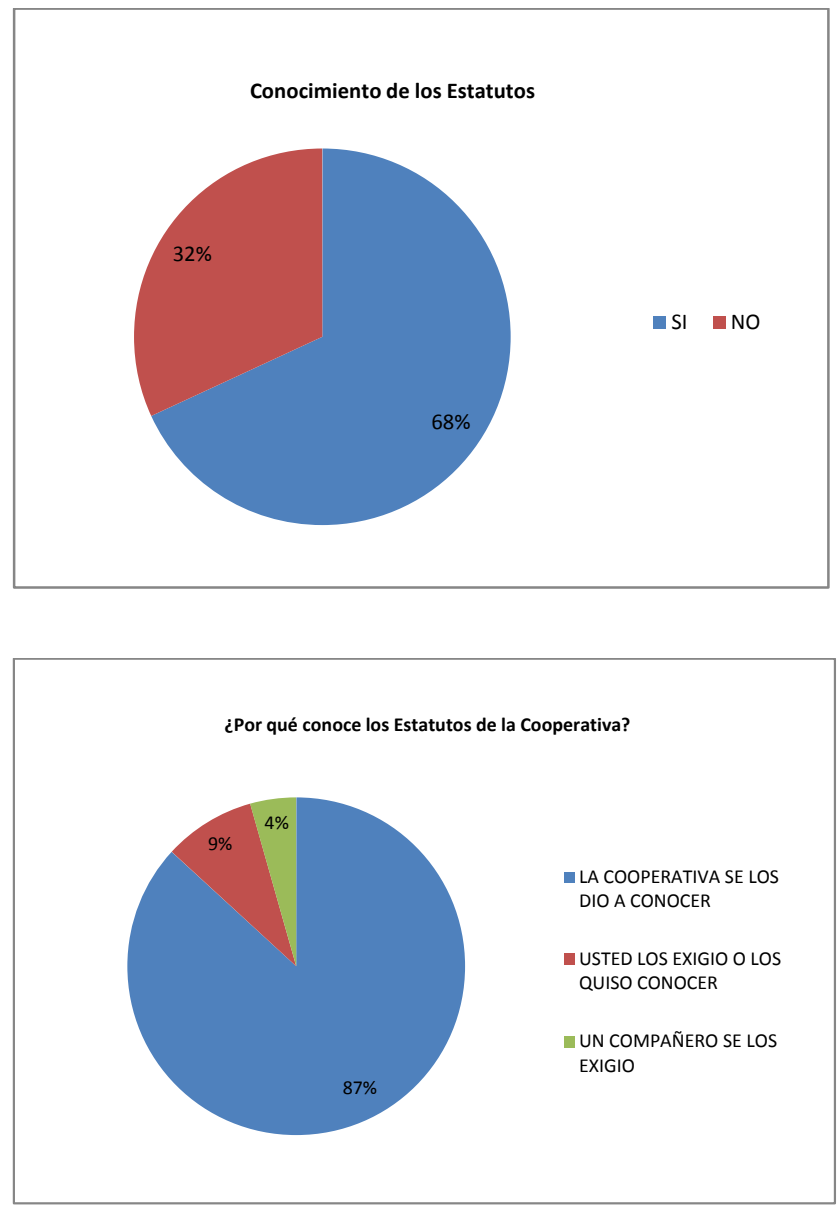

Fuente: la autora

Frente a los materiales para el cabal cumplimiento de las actividades de los asociados se puede concluir que en la mayoría de las oportunidades es la empresa donde prestan sus servicios la que los suministra. En cuanto a la realización de actividades de proyección social se encontró que las CTA en Bucaramanga se enfocan hacia aquellas referidas a la convivencia y actividades culturales. 
Figura 15. Naturaleza de las actividades de proyección social

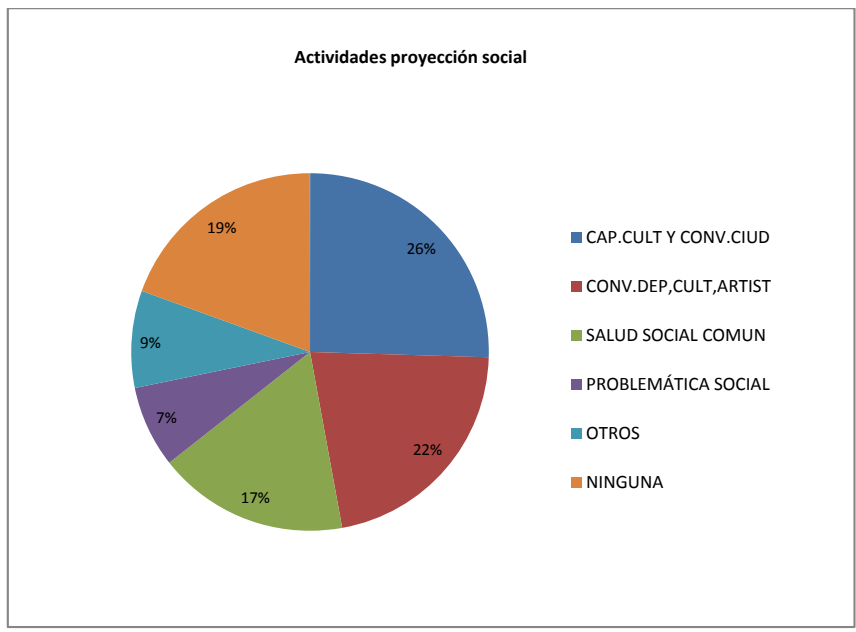

Fuente: la autora

\section{DISCUSIÓN}

Como bien se ha reiterado las CTA son asociaciones encargadas de vincular sujetos a través de un contrato de sociedad, basándose en el principio de la autonomía de la voluntad a través del cual se crean relaciones jurídicas que generan derechos y obligaciones inter-partes a través de un compromiso recíproco las partes se obligan mediante el contrato de sociedad con el fin de regular sus intereses, surgiendo como única limitante el respeto a las normas de orden público y la sana costumbre, para que una vez establecida la expresión de la voluntad ésta se convierta en ley para las partes y sólo pueda dejarse sin efectos por mutuo acuerdo o por causas legales.

Éstas, a su vez, son guiadas por estatutos en pro del cumplimiento de un objetivo social, que no es más que la prestación de servicios, ejecución de obras y producción de bienes específicos y especializados, cuya función debe ser desempeñada por un tercero que asume los riegos profesionales y la seguridad social, al actuar como dueño y trabajador de su misma empresa. El aporte principal como socio es su mano de obra y una porción de la compensación recibida como resultado de la labor desempeñada, debido a que es una asociación sin ánimo de lucro que se solidifica por medio del capital social. Sin embargo, según lo establecido por la Sentencia C-211 de 2000 como contraprestación por el cumplimiento de sus funciones, el asociado recibe cierta cantidad de dinero para su sustento y manutención básica. 
Estas organizaciones, en ejercicio del derecho de libre asociación, brindan ayuda frente a la disminución de la informalidad laboral al dar cumplimiento a lo dispuesto en el artículo 25 Constitución Política. Es por ello, que las CTA contribuyen con la restricción del abuso de la posición dominante, en ocasiones, ejercida por empleadores que se fructifican del trabajo de sus empleados sometiéndolos a jornadas extenuantes, exceso de cargas laborales y bajas remuneraciones, situación que incentiva a los trabajadores a crear asociaciones donde sean ellos mismos quienes impongan el desarrollo de sus labores y presten servicios bajo el principio de la participación, donde el aporte de ideas es esencial para su funcionamiento, creando así una organización cooperativa.

Por otro lado, el empresario (del sector social, colectivo o gremial) se ve beneficiado con la clase de contrato laboral que adquiere con las cooperativas, debido a que se exonera de pagos laborales que debiera efectuar en caso de tener trabajadores bajo la modalidad de contrato de trabajo, ya que éste deriva obligaciones que sólo representan gastos al empleador y que le causan un detrimento desde el punto de vista de sus beneficios, al utilizar recursos que pueden ser invertidos en actividades propias para obtener una inversión rentable que le otorgue intereses económicos. Por ello las CTA generan un beneficio al concederle al contratante un ahorro económico dado que sólo deben cancelar a la asociación por la prestación del servicio, la cual se encarga de hacer los pagos a sus asociados y realizar los descuentos pertinentes a seguridad social, riesgos profesionales, administración, retención en la fuente y aportes, entre otros.

De acuerdo a lo anterior, este factor influye en el campo laboral al promover la proliferación de estas asociaciones por parte de empleadores que crean cooperativas con el fin de vincular mano de obra a un bajo precio, situación que vulnera los derechos contemplados en la legislación laboral, debido a que los trabajadores asociados realizan las mismas labores que efectúan aquellos que son vinculados mediante contrato de trabajo, razón suficiente para aplicar las normas del derecho laboral colombiano (Código Laboral) que con base al principio de realidad sobre la forma impide la existencia de todo tipo de desigualdad laboral al no tener presente una proporcionalidad objetiva en las labores realizadas y ajustar a derecho los contratos, que en todo caso serán siempre a favor del trabajador en concordancia con el principio de favorabilidad.

No obstante, se puede evidenciar que en su mayoría las CTA han sido creadas por los mismos empleadores con el propósito de vincular personal o particulares para que realicen funciones sin contar con los medios o perfiles necesarios para desempeñar la labor por la cual fueron contratados, debido a que ejercen como intermediarios y/o EST, que obtienen beneficios de la mano de obra del asociado. Por tales razones, se considera que se atenta contra el principio de legalidad, puesto que pese a la existencia de regulación, su ejercicio no es conforme a la ley y su actuar ocasiona variaciones en la modalidad del contrato, que genera una oposición a los principios 
e interpretación constitucional y legal, que tienen como fin amparar los derechos vulnerados de los cooperados.

Sin embargo, el Gobierno Nacional y el Ministerio de Protección Social han unificado esfuerzos con el fin de crear normas que protejan los principios constitucionales vulnerados por las CTA y los empleadores al vincular de forma temporal a sus asociados, y omitir las exigencias legales requeridas.

Por tal razón, de acuerdo a los resultados que arroja el trabajo investigativo desarrollado, se puede concluir que aunque la intención del legislador y del Estado es generar empleo mediante esta figura, la realidad confirma que estas clases de asociaciones se han convertido en una forma más de evadir las responsabilidades patronales y prestacionales, lo cual va en contravía de los fines que busca la institucionalidad.

Cuando se promovió la creación y reglamentación de las CTA se determinó que "sus actividades deben cumplirse con fines de interés social y sin ánimo de lucro", sin embargo, la mayor parte de las CTA que funcionan en Bucaramanga, si bien se encuentran registradas en la Cámara de Comercio como tal figura, en realidad son empresas de servicios temporales o simplemente empresas de carácter comercial, puesto que su objeto no es producir o distribuir conjunta y eficientemente bienes o servicios para satisfacer las necesidades de sus asociados y de la comunidad en general, sino lucrarse mediante el trabajo de quienes ellos denominan "asociados".

Al aplicar las encuestas se encontró que una proporción significativa de las empresas que funcionan bajo esta figura asociativa, actúan como intermediarias laborales, al desnaturalizar asi la finalidad y objeto de las CTA y vulnerar las disposiciones constitucionales y de ley que les prohíbe taxativamente actuar como empresas de servicios temporales. Lo anterior con fundamento en que para los empresarios existen grandes beneficios; en la exposición de motivos del proyecto de la Ley 260 de 2005, se señaló que las empresas ahorran entre un 12 y 15\% en el pago a empresas de intermediación laboral, además de un $9 \%$ en pago de parafiscales y un 4\% en administración de nómina, lo anterior según el Boletín número 15 de 2007 del Observatorio de Mercado Laboral de la Universidad Externado de Colombia. Entre otros aspectos benéficos para los empresarios se encuentran los referentes a la exención del pago de indemnizaciones por despido injusto y la descarga en materia de obligaciones sindicales, pues al ser el asociado dueño de su propia empresa de trabajo, las empresas en las que ejercen sus actividades no cumplen la función de empleador.

Las CTA en Santander ocupan un sitio importante en el empleo regional y especialmente en Bucaramanga, lo cual incide en el dinamismo actual del mercado laboral del Área Metropolitana. No obstante, mediante la investigación se encontró que existen dos tipos o modalidades de CTA; aquellas que cumplen realmente con el objeto para el que han sido creadas y aquellas que son creadas como una fachada para evadir obligaciones laborales de los empleadores frente a sus empleados, puesto que su funcionamiento interno diverge tanto de su configuración legal como su 
configuración ideológica, lo cual afecta directamente al trabajador y vulnera los derechos inherentes al mismo.

En el primer tipo de Cooperativas el asociado es miembro activo en la conformación de las mismas, aportante y gestor, propio dueño de la CTA y no experimenta subordinación laboral ni los riesgos que ella implica ${ }^{7}$. La afiliación a la seguridad social ${ }^{8}$ es importante para los asociados, lo que garantiza el goce de sus derechos en materia de salud, riegos y pensiones, en las condiciones dignas y justas, es por ello que una de las exigencias del Gobierno Nacional, es precisamente el cumplimiento de las garantías a los asociados so pena de incurrir en sanciones.

La otra cara de las CTA, aquellas creadas precisamente para que las empresas se eximan de sus obligaciones laborales ${ }^{9}$, en lo que a ello atañe, la legislación laboral ha sido directa al señalar la procedencia de acciones, para contrarrestar los abusos a sus asociados, como quiera que se demuestre la existencia de los elementos propios de una relación laboral, al configurarse, lo que conoce la Corte como "contrato realidad".

Por otro lado, existen empresas y medios de producción que utilizan las CTA para disminuir costos, al obtener la tenencia o el manejo de los mismos, sin establecer convenios a través de la suscripción de un contrato civil o comercial según lo exige la Ley 1233 de 2008. Lo anterior conlleva a que muchas CTA suplanten las funciones de las EST y acudan a comentar que la empresa donde ejecutan los servicios les ha dado en comodato la utilización de los activos fijos y herramientas. Estos activos fijos necesitan que figuren como bienes entregados en comodato, puesto que no serán reconocidos a través de una depreciación sino a través de una amortización. Lo delicado de este punto es que si los activos son utilizados por una CTA para poder desarrollar el trabajo que luego facturaría dicha CTA, la amortización del activo ya no sería un gasto necesario en la contabilidad de la empresa que los entregó en comodato pues el activo no ayuda a generar los ingresos de esa empresa sino los de la CTA y, en ese caso, se pierde la relación de causalidad y necesidad que exige

$7 \quad$ Puesto que en caso de un conflicto, no sería aplicable la legislación ordinaria laboral puesto que de común acuerdo sus miembros acogen en sus estatutos los medios propicios para dar solución a sus conflictos

8 Anteriormente las cooperativas que estuvieran dentro de la clase de "trabajo asociado", podían determinar como mejor les pareciera, la forma en que sus miembros pagaran la seguridad social, mientras que los otros tipos de cooperativas debían obligatoriamente vincular a sus miembros a un régimen de salud.

9 Por ello se dispuso que tales CTA también tendrían que liquidar los aportes al ICBF, SENA y Caja de compensación (ver inciso final del art.1 del Decreto 2996 de septiembre de 2004), a pesar de que la ley que regula tales aportes (leyes 21 de1982 y 89 de 1988) sólo exige que los mismos se liquiden en el caso de los asalariados. Pero tal norma luego fue declarada nula por el Consejo de Estado en Sentencia de octubre 12 de 2006 y nuevamente, desde dicha fecha, las CTA están exoneradas de liquidar tales aportes (sólo los liquidarían en forma "voluntaria" pero no "obligatoria") 
el artículo 107 del Estatuto Tributario para que un gasto pueda ser aceptado como deducible fiscalmente ${ }^{10}$.

Por tales razones es habitual, entonces, que en algunas empresas de Bucaramanga se incorporen empleados a través de las CTA, pero algunas de ellas empiezan a funcionar independiente de los principios de solidaridad, cuando son manejadas por grupos donde no hay la intervención de los trabajadores y tampoco hacen parte de los procesos de elección de cuerpos directivos. Todo lo anterior hace que las nuevas formas de contratación disminuyan la calidad de vida de los trabajadores de la ciudad de Bucaramanga en vez de mejorarla.

\section{REFERENCIAS}

Boletín del Observatorio de Mercado laboral No. 15. Cooperativas de Trabajo asociado. Bogotá Universidad Externado de Colombia. 2007)

Buchelli, M. 2001. Desarrollo local y cooperativismo: el caso de la experiencia del Secretariado de Pastoral Social de la Diócesis de Socorro y San Gil, Departamento de Santander, Colombia. Recuperado de http://www.saber.ula.ve/bitstream/ 123456789/18610/1/articulo2-2.pdf el día 11 de agosto de 2009.

Circular externa 005 de 2007 y ¿Circular externa 005 de 2007? [Procuraduría General de la Nación].

Constitución Política de Colombia. Artículo 25. Gaceta Constitucional No. 116 de 20 de julio de 1991.

Corte Constitucional. Sentencia C-211 de 2000.

Decreto 1333 de 1989. Establece el régimen de constitución, reconocimiento y funcionamiento de las pre-cooperativas. Junio 21 de 1989. Diario Oficial No. 38.867

Decreto 1598 De 1963 [Con fuerza de Ley]. Actualiza legislación cooperativa.

Decreto 2879 de 2004. Medidas para controlar la evasión y elusión de aportes parafiscales y se dictan disposiciones en materia de Cooperativas y Pre-cooperativas de Trabajo Asociado. Septiembre 07 de 2004. Diario Oficial 45.665.

Decreto 468 de Febrero 23 de 2006. Por el cual se reglamentan las normas correspondientes a las cooperativas de trabajo asociado contenidas en la Ley 79 de 1988 y se dictan otras disposiciones sobre el trabajo cooperativo asociado. Diario Oficial No 39.201.

Decreto 4588 de Diciembre 27 de 2006, Por el cual se reglamenta la organización y funcionamiento de las Cooperativas y Pre-cooperativas de Trabajo Asociado.

10 La DIAN rechazaría como no deducible ese "gasto amortización" de los bienes entregados en comodato (comparece con lo indicado en los artículos 127 y 128 del Estatuto Tributario y con lo dicho por la DIAN en el concepto 70314 de septiembre 29 de 2005).

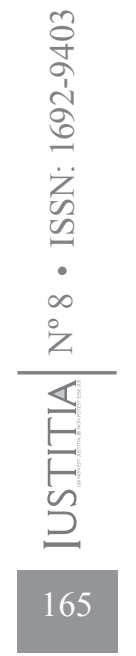


Díaz, L. (2005). Relaciones laborales Especiales: Las Empresas de Trabajo temporal y las Cooperativas. ¿Qué pueden hacer los sindicatos? Revista de Ciencias Sociales. Recuperado de http://redalyc.uaemex.mx/src/inicio/ArtPdfRed. jsp?iCve=28011109 Universidad de Zulia, Venezuela.

Fempopular, s.f. Cooperativismo en el mundo. Recuperado de Gómez, R (2008). Las Cooperativas de Trabajo Asociado en la intermediación laboral. Tesis de grado para optar por el título de Economista. [No publicada]. Abril de 2009.

Guerrero, A; Tristancho, I; Cediel, M (2005). Historia oral del sindicalismo en Santander. Colección Temas y Autores Regionales. Bucaramanga, Universidad Industrial de Santander; Escuela de Historia; Financiera Comultrasan, agosto 2005. 319- 404p. - (1 Ed.).

León, D (2007) Nuevas formas de contratación disminuyen la calidad de vida [Entrevista a Wilson Pardo Estévez]. Periódico 15 - Universidad Autónoma de Bucaramanga. Recuperado de http://www.universia.net.co/index2.php?option=com content\&do_pdf=1\&id=6648 el día 12 abril de 2009 .

Ley 100 de 1993. Sistema de seguridad social integral y se dictan otras disposiciones. Diciembre 23 de 1993 Diario Oficial número 41.148.

Ley 1111 de 2006. Modifica el estatuto tributario de los impuestos administrados por la dirección de impuestos y aduanas nacionales. Diciembre 27 de 2006. Diario Oficial 46.494.

Ley 1233 de 2008. Se precisan los elementos estructurales de las contribuciones a la seguridad social, se crean las contribuciones especiales con destino al SENA, al ICBF y a las Cajas de Compensación Familiar, se fortalece el control concurrente y se dictan otras disposiciones. Julio 22 de 2008. Diario Oficial 47.058

Ley 134 de 1931. Sobre sociedades cooperativas. Diciembre 07 de 1931. Diario Oficial No 21.866.

Ley 24 de 1981, por la cual se transforma la Superintendencia Nacional de Cooperativas en Departamento Administrativo Nacional de Cooperativas. Febrero 24 de 1981. Diario Oficial No. 35.711.

Ley 260 de febrero 07 de 2005. Por medio del cual se adiciona el Decreto 255 del 11 de febrero de 1992 y se dictan otras disposiciones. Diario oficial 45.816.

Ley 454 de Agosto 04 de 1998. Se determina el marco conceptual que regula la economía solidaria, se crea el Departamento Administrativo Nacional de la Economía Solidaria, la Superintendencia de la Economía Solidaria, el Fondo de Garantías para las Cooperativas Financieras y de Ahorro y Crédito, se dictan normas sobre la actividad financiera de las entidades de naturaleza cooperativa y se expiden otras disposiciones. Agosto 04 de 1998. Diario oficial 43.357.

Ley 50 de 1990. Introducen reformas al Código Sustantivo del Trabajo y se dictan otras disposiciones. Enero 1 de 1991. Diario Oficial No. 39.618.

Ley 79 de 1988, por el cual se actualiza la legislación cooperativa. Diciembre 23 de 1988. Diario oficial 38.648. 
Portal cooperativo. Historia del cooperativismo en Colombia. Recuperado de el día 17 de mayo de 2009.

Razeto, L. (1997). Los caminos de la economía de solidaridad.

Restrepo, V (1895). Los chibchas antes de la conquista española. Edición original: Bogotá, Imprenta de la luz. Recuperado de http://www.lablaa.org/blaavirtual/ historia/ chibch/indice.htm el día 2 de julio de 2009.

Rojas, A (2004). La intermediación laboral. Revista de Derecho, Universidad del Norte, número 22, pp. 167-210. Recuperada de http://ciruelo.uninorte.edu.co/pdf/ derecho/ 22/8_LA\%20INTERMEDIACION\%20LABORAL_DERECHO_No\%20 22.pdf el día 11 de junio de 2009.

Superintendencia de Economía Solidaria (2007). Base de datos Cooperativas. Recuperado de http://www.supersolidaria.gov.co/data/ CtasCamaraComercioPagWebverlfeb08. zip el día 1 de junio de 2009 


\section{APÉNDICE 1}

Tabla 1. Cooperativas y Pre-cooperativas en Colombia 2007

\begin{tabular}{lcc}
\hline Departamento & Número & $\%$ País \\
\hline Bogotá & 1761 & $14,60 \%$ \\
Valle & 1389 & $11,52 \%$ \\
Santander & 1246 & $10,33 \%$ \\
Atlántico & 1166 & $9,67 \%$ \\
Antioquia & 848 & $7,03 \%$ \\
Huila & 621 & $5,15 \%$ \\
Cauca & 474 & $3,93 \%$ \\
Nariño & 466 & $3,86 \%$ \\
Tolima & 464 & $3,85 \%$ \\
Cundinamarca & 448 & $3,72 \%$ \\
Cesar & 428 & $3,55 \%$ \\
N. de Sder. & 420 & $3,48 \%$ \\
Meta & 363 & $3,01 \%$ \\
Boyacá & 314 & $2,60 \%$ \\
Bolívar & 310 & $2,57 \%$ \\
Caldas & 284 & $2,36 \%$ \\
Córdoba & 204 & $1,69 \%$ \\
Risaralda & 200 & $1,66 \%$ \\
Quindío & 138 & $1,14 \%$ \\
Putumayo & 110 & $0,91 \%$ \\
Casanare & 78 & $0,65 \%$ \\
La Guajira & 72 & $0,60 \%$ \\
Sucre & 66 & $0,55 \%$ \\
Arauca & 40 & $0,33 \%$ \\
Caquetá & 38 & $0,32 \%$ \\
Choco & 29 & $0,24 \%$ \\
San Andrés & 19 & $0,16 \%$ \\
Magdalena & 14 & $0,12 \%$ \\
Guaviare & 12 & $0,10 \%$ \\
Amazonas & 8 & $0,07 \%$ \\
Guainía & 4 & $0,03 \%$ \\
Vichada & 3 & $0,02 \%$ \\
Total Nacional & 12.060 & \\
\hline & &
\end{tabular}

Superintendencia de Economía Solidaria (2007) 
Tabla 2. Cooperativas y Pre-cooperativas de Trabajo Asociado en Santander (2007)

\begin{tabular}{clcc}
\hline$\#$ & \multicolumn{1}{c}{ Municipio } & Número & $\%$ \\
\hline 1 & Bucaramanga & $\mathbf{6 3 7}$ & $\mathbf{5 1 , 1 2 \%}$ \\
$\mathbf{2}$ & Barrancabermeja & 129 & $10,35 \%$ \\
$\mathbf{3}$ & Puerto Wilches & 66 & $5,30 \%$ \\
$\mathbf{4}$ & Floridablanca & 61 & $4,90 \%$ \\
$\mathbf{5}$ & Girón & 49 & $3,93 \%$ \\
$\mathbf{6}$ & San Gil & 37 & $2,97 \%$ \\
$\mathbf{7}$ & Piedecuesta & 30 & $2,41 \%$ \\
$\mathbf{8}$ & Río Negro & 22 & $1,77 \%$ \\
$\mathbf{9}$ & Sabana De Torres & 14 & $1,12 \%$ \\
$\mathbf{1 0}$ & Barbosa & 13 & $1,04 \%$ \\
$\mathbf{1 1}$ & Charalá & 13 & $1,04 \%$ \\
$\mathbf{1 2}$ & Cimitarra & 13 & $1,04 \%$ \\
$\mathbf{1 3}$ & Málaga & 12 & $0,96 \%$ \\
$\mathbf{1 4}$ & San Vicente Chucuri & 12 & $0,96 \%$ \\
$\mathbf{1 5}$ & Landázuri & 11 & $0,88 \%$ \\
$\mathbf{1 6}$ & Puerto Parra & 11 & $0,88 \%$ \\
$\mathbf{1 7}$ & Mogotes & 8 & $0,64 \%$ \\
$\mathbf{1 8}$ & Vélez & 8 & $0,64 \%$ \\
$\mathbf{1 9}$ & Socorro & 7 & $0,56 \%$ \\
$\mathbf{2 0}$ & El Carmen & 5 & $0,40 \%$ \\
$\mathbf{2 1}$ & El Playón & 5 & $0,40 \%$ \\
$\mathbf{2 2}$ & Barichara & 4 & $0,32 \%$ \\
$\mathbf{2 3}$ & Guaca & 4 & $0,32 \%$ \\
$\mathbf{2 4}$ & Lebrija & 4 & $0,32 \%$ \\
$\mathbf{2 5}$ & Onzaga & 4 & $0,32 \%$ \\
$\mathbf{2 6}$ & Páramo & 4 & $0,32 \%$ \\
$\mathbf{2 7}$ & San Joaquín & 4 & $0,32 \%$ \\
$\mathbf{2 8}$ & Suaita & 4 & $0,32 \%$ \\
$\mathbf{2 9}$ & Tona & 4 & $0,32 \%$ \\
\hline & & & \\
\hline
\end{tabular}

\begin{tabular}{llrr}
\hline$\#$ & \multicolumn{1}{c}{ Municipio } & Número & \multicolumn{1}{c}{$\%$} \\
\hline $\mathbf{3 0}$ & Betulia & 3 & $0,24 \%$ \\
$\mathbf{3 1}$ & Pinchote & 3 & $0,24 \%$ \\
$\mathbf{3 2}$ & Santa Bárbara & 3 & $0,24 \%$ \\
$\mathbf{3 3}$ & Valle De San José & 3 & $0,24 \%$ \\
$\mathbf{3 4}$ & Aratoca & 2 & $0,16 \%$ \\
$\mathbf{3 5}$ & California & 2 & $0,16 \%$ \\
$\mathbf{3 6}$ & Cerrito & 2 & $0,16 \%$ \\
$\mathbf{3 7}$ & Confines & 2 & $0,16 \%$ \\
$\mathbf{3 8}$ & Encino & 2 & $0,16 \%$ \\
$\mathbf{3 9}$ & Florián & 2 & $0,16 \%$ \\
$\mathbf{4 0}$ & Guapota & 2 & $0,16 \%$ \\
$\mathbf{4 1}$ & Los Santos & 2 & $0,16 \%$ \\
$\mathbf{4 2}$ & Matanza & 2 & $0,16 \%$ \\
$\mathbf{4 3}$ & Ocamonte & 2 & $0,16 \%$ \\
$\mathbf{4 4}$ & Oiba & 2 & $0,16 \%$ \\
$\mathbf{4 5}$ & Palmas Socorro & 2 & $0,16 \%$ \\
$\mathbf{4 6}$ & San Andrés & 2 & $0,16 \%$ \\
$\mathbf{4 7}$ & San José de Miranda & 2 & $0,16 \%$ \\
$\mathbf{4 8}$ & Simacota & 2 & $0,16 \%$ \\
$\mathbf{4 9}$ & Bolívar & 1 & $0,08 \%$ \\
$\mathbf{5 0}$ & Cabrera & 1 & $0,08 \%$ \\
$\mathbf{5 1}$ & Concepción & 1 & $0,08 \%$ \\
$\mathbf{5 2}$ & Coromoro & 1 & $0,08 \%$ \\
$\mathbf{5 3}$ & Gambita & 1 & $0,08 \%$ \\
$\mathbf{5 4}$ & Güepsa & 1 & $0,08 \%$ \\
$\mathbf{5 5}$ & Hato & 1 & $0,08 \%$ \\
$\mathbf{5 6}$ & La Paz & 1 & $0,08 \%$ \\
$\mathbf{5 7}$ & Puente Nacional & 1 & $0,08 \%$ \\
Total En Santander & $\mathbf{1 2 4 6}$ \\
\hline & & & \\
\hline
\end{tabular}

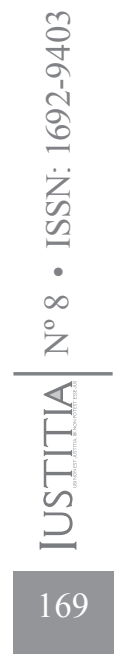

